Document downloaded from:

http://hdl.handle.net/10251/52699

This paper must be cited as:

Lloréns Rodríguez, R.; Naranjo Ornedo, V.; López-Mir, F.; Alcañiz Raya, ML. (2012). Jaw tissues segmentation in dental 3D CT images using fuzzy-connectedness and

morphological processing. Computer Methods and Programs in Biomedicine. 108(2):832843. doi:10.1016/j.cmpb.2012.05.014.

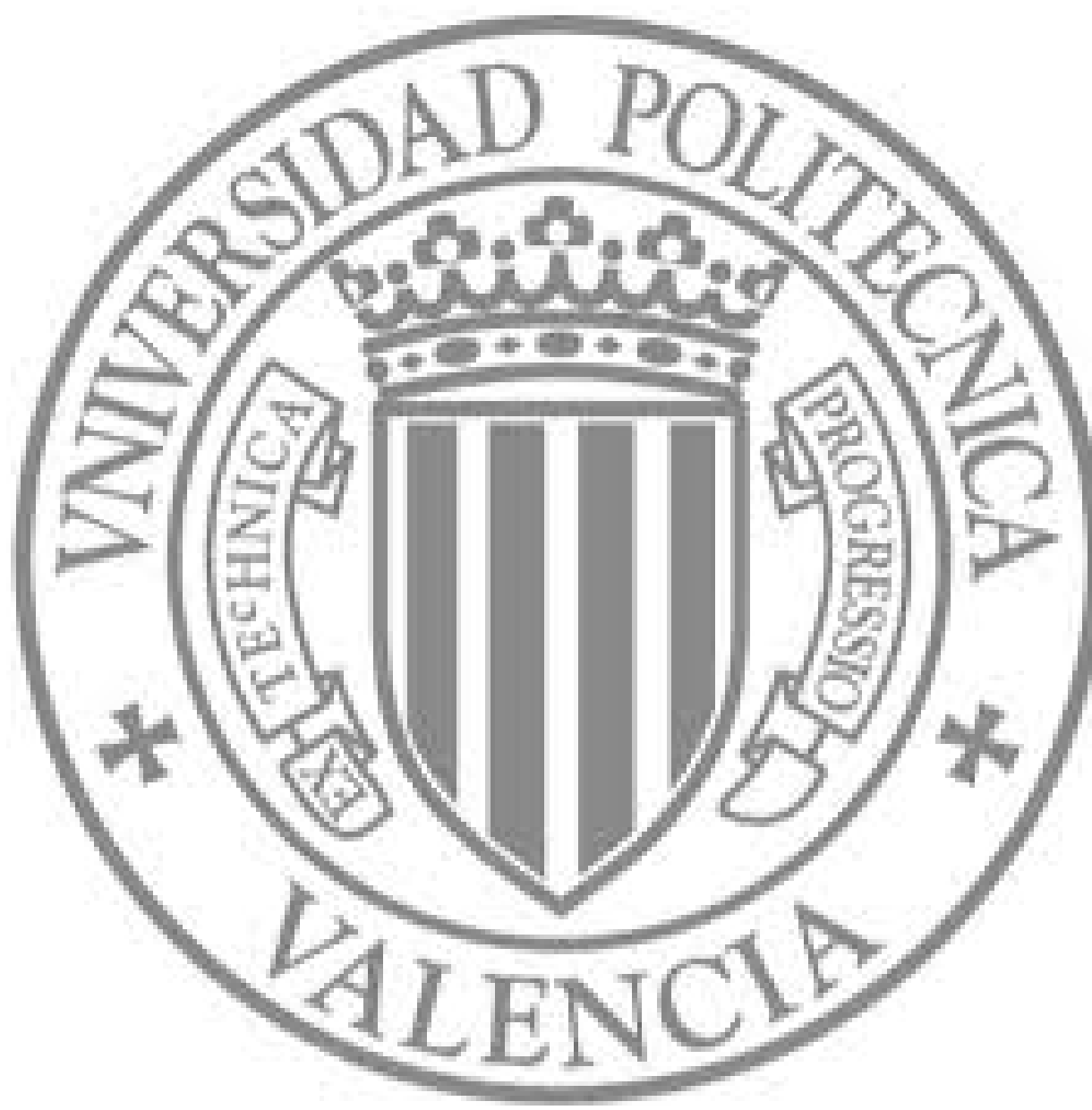

The final publication is available at

http://dx.doi.org/10.1016/j.cmpb.2012.05.014

Copyright Elsevier 


\title{
Jaw tissues segmentation in dental 3D CT images using fuzzy-connectedness and morphological processing
}

\author{
Roberto Lloréns ${ }^{\mathrm{a}}$, Valery Naranjo ${ }^{\mathrm{a}}$, Fernando López ${ }^{\mathrm{a}}$, Mariano Alcañiz ${ }^{\mathrm{a}, \mathrm{b}}$ \\ ${ }^{a}$ Instituto Interuniversitario de Investigación en Bioingeniería y Tecnología Orientada al \\ Ser Humano, Universitat Politècnica de València \\ Camino de Vera s/n, 46022 Valencia, Spain. \\ ${ }^{b}$ Ciber, Fisiopatología Obesidad y Nutrición, CB06/03, Instituto de Salud Carlos III \\ Av. Sos Baynat s/n, Univesity of Jaume I, 12071 Castellón, Spain \\ email: rllorens@labhuman.i3bh.es ${ }^{1}$
}

\begin{abstract}
The success of oral surgery is subject to accurate advanced planning. In order to properly plan for dental surgery or a suitable implant placement, it is necessary an accurate segmentation of the jaw tissues: the teeth, the cortical bone, the trabecular core and over all, the inferior alveolar nerve. This manuscript presents a new automatic method that is based on fuzzy connectedness object extraction and mathematical morphology processing. The method uses computed tomography data to extract different views of the jaw: a pseudo-orthopantomographic view to estimate the path of the nerve and cross-sectional views to segment the jaw tissues. The method has been tested in a groundtruth set consisting of more than 9000 cross-sections from 20 different patients and has been evaluated using four similarity indicators (the Jaccard index, Dice's coefficient, point-to-point and point-tocurve distances), achieving promising results in all of them $(0.726 \pm 0.031$, $0.840 \pm .019,0.144 \pm 0.023 \mathrm{~mm}$ and $0.163 \pm 0.025 \mathrm{~mm}$, respectively). The method has proven to be significantly automated and accurate, with errors around $5 \%$ (of the diameter of the nerve), and is easily integrable in current dental planning systems.
\end{abstract}

Keywords: jaw tissue segmentation/reconstruction, inferior alveolar nerve, automatic computer-aided surgery, fuzzy connectedness

\footnotetext{
${ }^{1}$ This work has bee supported by the project MIRACLE (DPI2007-66782-C03-01AR07) of Spanish Ministerio de Educación y Ciencia.
} 


\section{Introduction}

The lower jaw is the densest and most prominent bone in the face. It is made up of four easily distinguishable tissues: the teeth, a hard exterior cortical bone that contains a softer osseous tissue that is filling its inner cavity, the trabecular (or cancellous or spongy) core, and the mandibular canal (when present), which contains the inferior alveolar nerve. The nerve, together with the inferior alveolar artery, constitutes the inferior alveolar neurovascular bundle. The inferior alveolar nerve runs along the lower jaw, crossing both hemimandibles, from mandibular foramen to mental foramen through the inferior alveolar canal, providing sensation to the mandible. For this reason, an injury to the canal might result in temporary or permanent numbness and paresthesia. Consequently, the nerve must be avoided in any type of surgical intervention. All this gives rise to the need for accurate segmentation that provides precise information to assure the success of the dental surgery for a great number of medical applications such as dental implant planning systems, plastic reconstructive surgery, etc.

Many dental applications carry out the process of 3D reconstruction from CT data de-emphasizing tissue segmentation as in [1], and many others delegate this task to dentists or surgeons, providing tools for this purpose [2]. Fütterling et al. [3] carry out a segmentation of hard tissues by thresholding, while inner tissues are segmented by assigning different material properties to the tetrahedral finite elements, depending on the density values in the CT data-set. Kršek et al. [4] present a tissue segmentation process that requires a high level of human interaction that is assisted only by basic morphological operations and thresholding in Hounsfield values. Xiaojun et al. [5] threshold the CT data in order to segment the hard tissues and use a semi-automatic region growing method to find the contours of the inferior alveolar nerve in cross-sections, which are finally closed in a bicubic B-spline. Focusing on the segmentation of the inferior alveolar nerve, Stein et al. [6] use Dijkstra's algorithm aided by 3D morphology to trace the most favorable path between two nodes (here, the mandibular foramen and mental foramen) marked by an expert. Similarly, Kondo et al. [7] use a panoramic projection (which mostly includes the nerve) inverting and multiplying it by its second derivative in order to isolate this tissue. The nerve is then bidirectionally tracked starting from two nodes (specified manually) using a mask-based algorithm. Rueda 
et al. [8] use active appearance models (AAM) for the segmentation of jaw tissues in serial cross-sections of the jaw, but the precision achieved is insufficient. Recently, Kainmuller et al. [9] combine a statistical model of the nerve with an optimization of Dijkstra's algorithm.

These methods are either not accurate enough or require several human interactions. Lloréns et al. [10] evaluated and validated the fuzzy connectedness methodology presented by Udupa [11, 12] for the segmentation of jaw tissues achieving encouraging results. Our aim is to adapt the work of Lloréns et al. to design a method that allows the accurate segmentation and 3D reconstruction of jaw tissues. Kim et al. [13] presented a very similar approach but using a variation of Dijkstra's algorithm.

\section{Method}

The method presented in this paper aims to reconstruct the tissues of the human jaw from a set of serial cross-sections that are defined perpendicularly to the dental arch plane (section 2.1). The jaw is divided into 5 areas according to the tissues present in them. The method scans the jaw slice by slice. The algorithm estimates which area each cross-section belongs to and attempts to segment the tissues that are present (section 2.3). The hard tissues, it is the cortical bone and the teeth, are segmented by thresholding in Hounsfield domain. The spongy bone is defined as the inner area of the cortical bone that is not considered to be as part of the nerve. The nerve is segmented by means of the fuzzy connectedness object extraction algorithm (FCOE), so it is necessary to know at least the position of one pixel belonging to this tissue to play the part of a seed (section 2.2). With this in mind, a pseudo-orthopantomographic projection is built, as described below (subsection 2.1), where a wider view of the nerve can be observed. In this view, the nerve is fitted by means of a second-degree function since the nerve describes an almost quadratic trajectory. This provides a seed for the segmentation method. These projections also allow those cross-sections where the segmentation of the nerve may be carried out with high probability of error to be determined. In these cases, and in those where the segmentation is wrong, only the hard tissues are segmented, and the nerve and trabecular bone are predicted later using an interpolation technique based on Fourier descriptors (section 2.4). When all the cross-sections are segmented, the 3D volume of the different tissues is reconstructed by means of the marching cubes algorithm (section 2.5). 


\subsection{Materials}

This section details the materials and tools at our disposal, as well as the way they were obtained. The CT data was provided by GE Medical Systems HiSpeed QXi and Philips Medical Systems - Philips CT Aura. CT volumes are comprised of slices of size $512 \times 512$ pixels separated by $1 \mathrm{~mm}$, with resolutions of $\Delta_{x}=\Delta_{y}=0.26 \mathrm{~mm}$ and $\Delta_{z}=1 \mathrm{~mm}$. The number of slices varies in each patient.

In order to reformat the CT data, Implametric software was used. Implametric (3Dent(C), Spain) is a dental implant planning system, that allows points along the dental arch to be defined in a transversal view as shown in figure 2.a to describe the dental arch curve. However, there are a great number of commercially available programs that allow this task to be carried out. In addition, this parabola can be estimated by means of morphologic operations as described in [7].

The cross-sectional set, denoted as $\Omega_{N}$, consists of a series of $\mathrm{N}$ slices or cross-sections that are orthogonally oriented to the dental arch plane and separated $0.20 \mathrm{~mm}$ from each other.

$$
\Omega_{N}=\left\{\text { section }_{1}, \text { section }_{2}, \ldots, \text { section }_{N}\right\}
$$

For example, figure 2.a shows a patient's mandible, whose dental arch length is $192.1 \mathrm{~mm}$. Consequently, the cross-sectional sets of both hemimandibles are made up of 480 slices. Cross-section number $233\left(\Omega_{480}^{233}\right)$ is shown in figure 2.b.

With regard to the size of the cross-sections, the width ( $\mathrm{x}$ axis in the coordinate system of figure 1) is 153 pixels and the height (y axis) depends on the number of slices of the CT data set.

The pseudo-orthopantomographic projection, denoted as $\Gamma^{n}$, is obtained from the half of the dental arch plane that corresponds to the hemimandible under study. Projection $\Gamma^{n}$ is made by concatenating the $n$-th column of all the $\mathrm{N}$ cross-sections of the set $\Omega_{N}$ in the following way:

$$
\Gamma^{n}(:, i)=\Omega_{N}^{i}(:, n)
$$

with i ranging from 1 to $\mathrm{N}$. Consequently, the width of the projections is $\mathrm{N}$ pixels and the height is the height of the cross-sections, as shown in figure 3.

The processing core of the algorithm was developed on Matlabß and the 3D reconstructing process was carried out by means of VTK libraries on Visual Studioß 2005. 


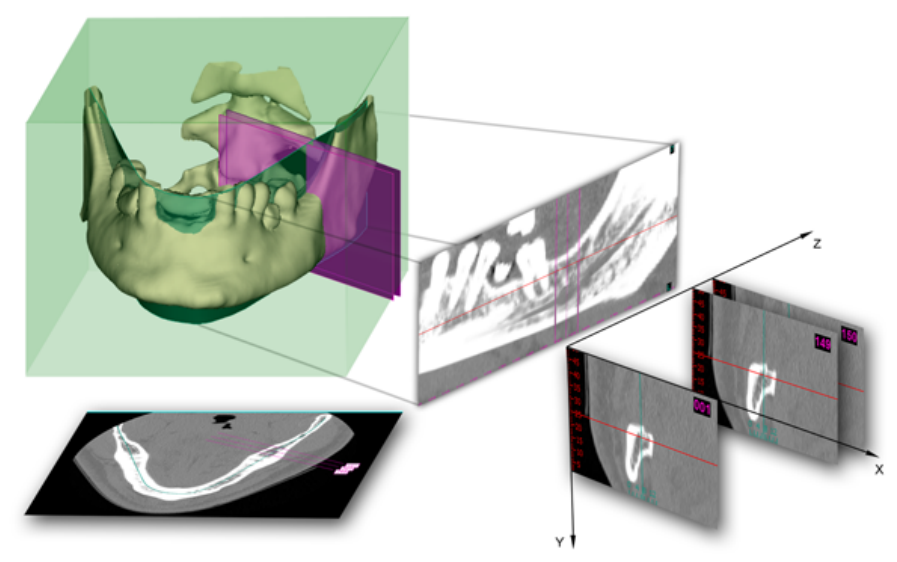

Figure 1: Acquisition of cross-sections and a pseudo-orthopantomographic projection from a CT volume.

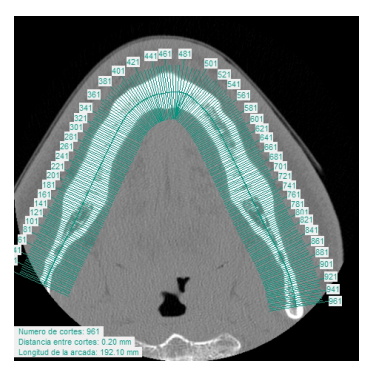

a)

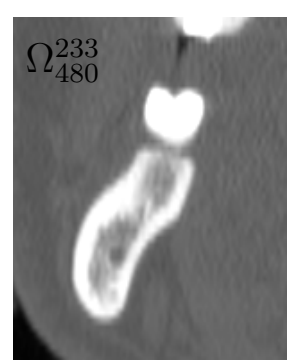

b)

Figure 2: a) CT slice where the dental arch curve has been defined; b) Example of a cross-section.

\subsubsection{Anatomical region labelling}

Since the extension of the nerve does not cover the whole jaw, there are cross-sections where the channel is not present, as in the chin. It is therefore necessary to know the tissues to be segmented in each slice. To fulfill this task, the buccal environment is divided into different areas.

- area 1. From the edge of the ramus to the mandibular foramen: this section includes the area from the beginning of the jaw up to the mandibular foramen. Only teeth, cortical bone and trabecular core are present. 


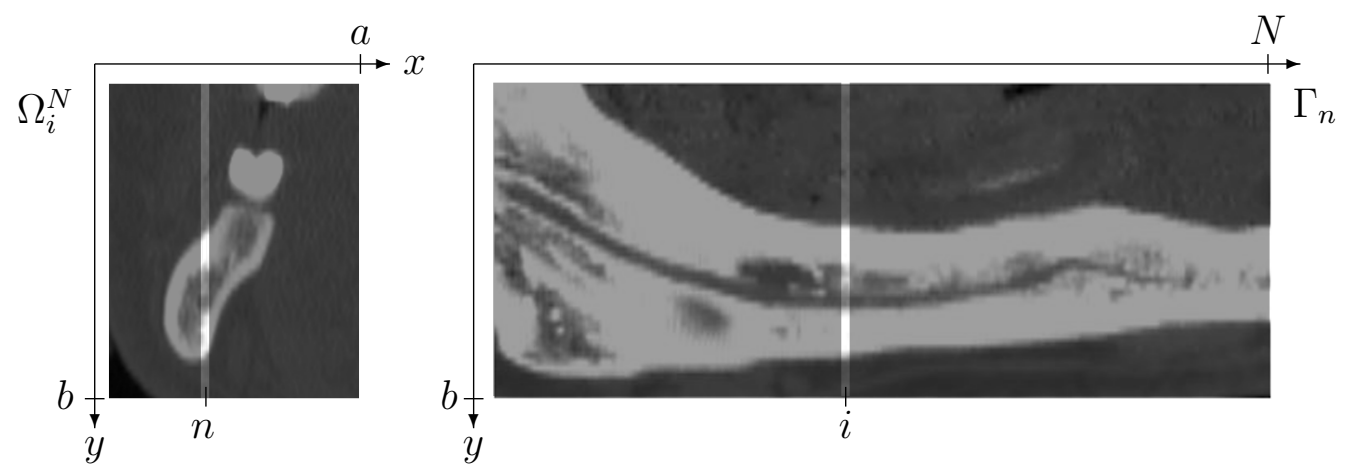

Figure 3: Construction of pseudo-orthopantomographic projections from cross-sections

- area 2. Mandibular foramen: the inferior alveolar nerve enters the mandible via the mandibular foramen and runs along the body of the jaw.

- area 3. Body: this section includes part of the ramus, the angle, and the mandibular body where the nerve is embedded in the mandibular canal within the mandible.

- area 4. Mental foramen: the nerve exits the mandible through the mental foramen, at about the second premolar.

- area 5. Mandibular symphysis: half of the parasymphyseal area.

Figure 4 shows the division of the jaw. An example of cross-sections of each area is included. As shown, the inferior alveolar nerve is only present in areas 2, 3 and 4 .

Due to the fact that cross-sections are processed sequentially depending on the area they belong to, it is important to define a solid algorithm in order to determine the change of area (where one area ends and the other area begins). The algorithm is based on the entrance and exit of the channel in both foramina to determine the change of area. For this reason, the region of interest is focused on these apertures. A mask is defined by computing the ellipse that closes the $2 \mathrm{D}$ shape of the section of the cortical bone in the crosssections. The major axis is computed, and regions $\alpha$ and $\beta$ are defined as the upper-right region and lower-left region, respectively, as shown in figure 5 . Specifically, the algorithm evaluates the break and recovery of the section of the cortical bone. Thus, while in area 1, if a break is detected in region 


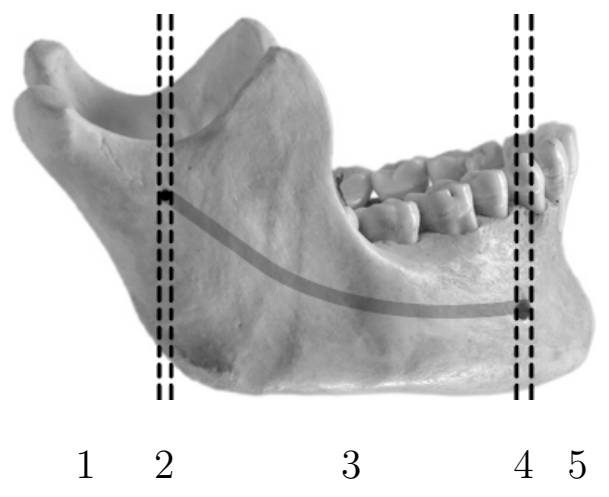

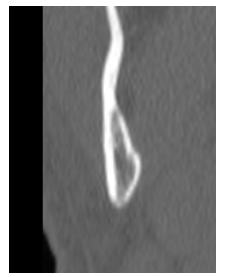

a)

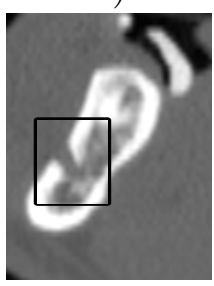

d)

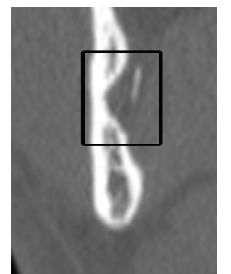

b)

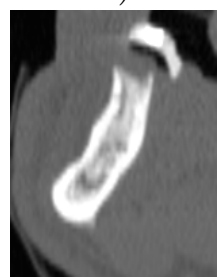

e)

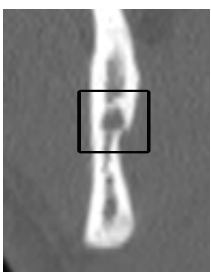

c)

Figure 4: a) Hemimandible division. Examples of a) area 1; b) area 2; c) area 3; d) area 4 ; e) area 5.

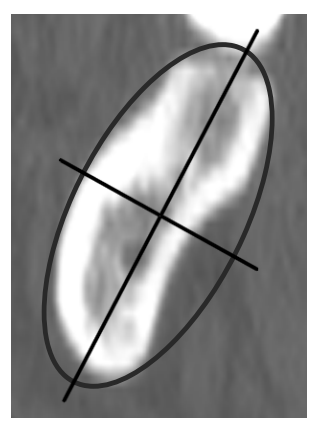

a)

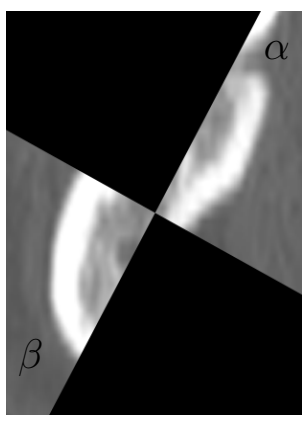

b)

Figure 5: a) Estimation of the ellipse closing the surface; b) Definition of the mask.

$\alpha$, the break indicates the entrance of the alveolar bundle into the jaw, and consequently the change into area 2 . However, while in area 2, if a recovery is detected in $\alpha$, the recovery indicates that the canal has finally entered into the jaw, which implies the change into area 3.

Similarly, from the region 3 onward, the algorithm evaluates the $\beta$ region. When the nerve begins to exit, a break in the cortical bone is detected, 
indicating the change into area 4. Finally, when the cortical bone is recovered, it implies the change into area 5.

The break and recovery of the cortical bone are detected by means of mathematical morphology processing as explained in algorithm 1.

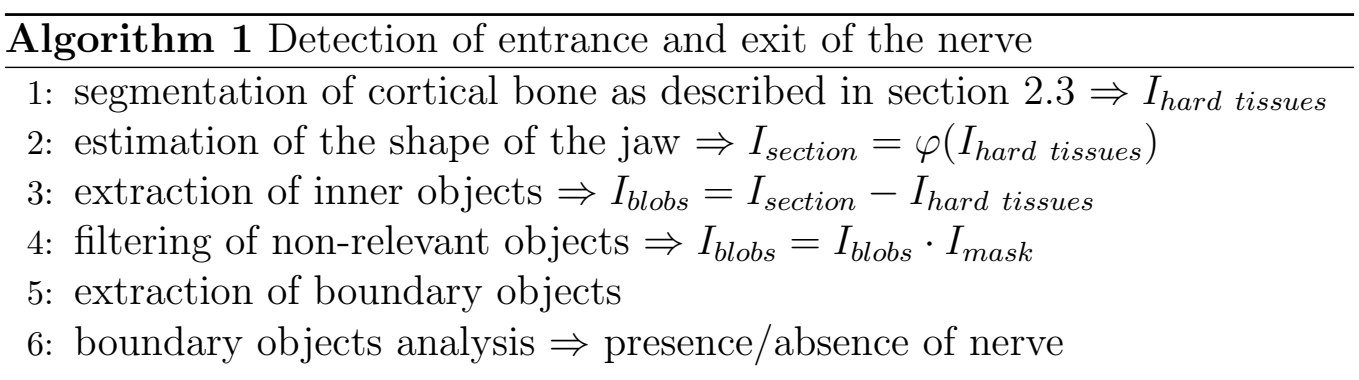

The expression $\varphi(x)$ represents the morphological closing of image $x$, which leads to the elimination of the darkest areas. As shown in the algorithm, the inner part of the cortical bone is analyzed. If any blob intersects the boundary with a large enough geometrical area, it is considered as an entrance or exit of the nerve. In contrast, the cortical bone is considered to be a continuous surface.

\subsection{Fuzzy connectedness fundamentals}

Fuzzy connectedness is a fuzzy subset theory-based methodology. The algorithm starts from a seed and evaluates the affinity between the seed and the pixels in the image, as explained in [11, 12]. Thus, the algorithm computes the connectivity map of the image under study, where each pixel value represents the affinity between the pixel and the seed. Consequently, it is intuitive to define an object as those pixels whose connectivity value is greater than a certain threshold.

The affinity describes the similarity between two pixels and represents the power of the connection between them. For this reason, the affinity is based on the adjacency between the pixels and on the similarity of their intensities. Adjacency represents the contiguity between pixels. For this study, 4-adjacency is considered and can be defined for pixels $c_{i}$ and $d_{i}$ as follows:

$$
\mu_{\alpha}(c, d)= \begin{cases}1 & \text {,if } \sqrt{\sum_{i}\left(c_{i}-d_{i}\right)^{2}} \leq 1 \\ 0 & \text {,otherwise }\end{cases}
$$

Analytically, the affinity can be expressed as:

$$
\mu_{\kappa}(c, d)=h\left(\mu_{\alpha}(c, d), f(c), f(d), c, d\right)
$$


That is, the affinity between the pixels depends on their adjacency, their position, as well as on some function of these two parameters.

According to the fuzzy connectedness theory described in [14], besides the adjacency, the affinity should consist of two components: an object-featurebased component and a homogeneity-based component. Both components must be considered in the design of the affinity, although in some applications, it is more productive to consider only one component.

Therefore we can design a great variety of functions for each component separately, and then combine them to obtain a desired affinity relation that is valid for the application under study. It is then possible to refine the affinity as follows

$$
\mu_{\kappa}(c, d)=\mu_{\alpha}(c, d) g\left(\mu_{\Psi}(c, d), \mu_{\Phi}(c, d)\right)
$$

where $\mu_{\Psi}$ and $\mu_{\Phi}$ represent the homogeneity-based component and the objectfeature-based component, respectively.

By defining $g, \mu_{\Psi}$ and $\mu_{\Phi}$, it is possible to generate an affinity family for any given fuzzy relation $\kappa, \mu_{\kappa}$. As described in [10], the best performance for the segmentation of jaw tissues is achieved with the configuration shown in table 1, where $M$ and $\sigma$ are the mean and the standard deviation and subindexes $o$ and $h$ refer to the intensities and the intensity differences (respectively) of the defined cortical and channel regions. All the parameters have been set as described below in table2.

$\mu_{\kappa}=\mu_{\alpha}\left(\left(1-\min \left(\mu_{\Psi}, \frac{1}{2} \mu_{\Phi}\right)\right) \mu_{\Phi}+\min \left(\mu_{\Psi}, \frac{1}{2} \mu_{\Phi}\right) \mu_{\Psi}\right)$
$\mu_{\Psi}=\left\{\begin{array}{ll|}1 & \text {,if } 0 \leq|f(c)-f(d)| \leq a_{1 \Psi} \\ \frac{a_{2 \Psi}-|f(c)-f(d)|}{a_{2 \Psi}-a_{1 \Psi}} & \text {,if } a_{1 \Psi} \leq|f(c)-f(d)| \leq a_{2 \Psi} \\ 0 & \text {,if }|f(c)-f(d)|>a_{2 \Psi}\end{array}\right.$
$\mu_{\Phi}=e^{-\frac{\left(\frac{f(c)+f(d)}{2}-m_{o}\right)^{2}}{2 k_{o}^{2}}}, k_{o}>0$

Table 1: Affinity components configuration

As mentioned above, the FCOE starts from a seed, which is a pixel belonging to the object, and computes the affinity of every pixel of the image with that seed. Therefore the correct estimation of the seed is critical since a wrong seed will lead to a wrong segmentation. To overcome this problem, a 


\begin{tabular}{|l|l|}
\hline$\mu_{\Psi}$ & $a_{\Psi}=M_{h}+t \sigma_{h}, a_{1 \Psi}=0, a_{2 \Psi}=M_{h}+t \sigma_{h}, k_{\Psi}=M_{h}+t \sigma_{h}$ \\
\hline$\mu_{\Phi}$ & $m_{o}=M_{o}, a_{o}=t \sigma_{o}, a_{1 o}=0, a_{2 o}=t \sigma_{o}, k_{o}=t \sigma_{o}$ \\
\hline
\end{tabular}

Table 2: Parameters description

new approach is presented using the maximum information available in the CT study.

Our approach is based on the fact that the trajectory of the nerve within the mandible describes a pseudo-quadratic path. Consequently, our aim is to fit that trajectory using a polynomial in order to obtain any point that belongs to the nerve (along its whole extension). To successfully carry out the fitting process the nerve must be isolated as much as possible.

Since the pseudo-orthopantomographic projections are separated by 0.26 $\mathrm{mm}$, the nerve can be viewed in several consecutive projections. In order to isolate it, three consecutive projections containing the nerve are thresholded and multiplied. The nerve has proven to have density values between 0 and $300 \mathrm{HU}$ in all our experimental studies, so it allows a coarse segmentation to be carried out. Figure 6.a shows examples of consecutive projections, which are denoted as $\left(\Gamma^{i-1}, \Gamma^{i}, \Gamma^{i+1}\right)$, and figure 6.b shows the resulting images after thresholding, which are denoted as $\left(\Gamma_{t h}^{i-1}, \Gamma_{t h}^{i}, \Gamma_{t h}^{i+1}\right)$. Fig.7.a shows that common areas in adjoining pseudo-orthopantomographic projections, like the nerve and other spongy tissues, remain after multiplication.

$$
\begin{gathered}
\Gamma_{t h}^{i}=\left\{\mathbf{x} \mid \Gamma^{i}(\mathbf{x})>0 \mathrm{HU}\right\} \cap\left\{\mathbf{x} \mid \Gamma^{i}(\mathbf{x})<300 \mathrm{HU}\right\} \\
\Gamma=\Gamma_{t h}^{i-1} \cap \Gamma_{t h}^{i} \cap \Gamma_{t h}^{i+1}
\end{gathered}
$$

To improve isolation, the top-hat operation [15] is applied on the resulting image. All the elements of the image that can be fitted in a vertical structuring element with size 10 pixels $(2.6 \mathrm{~mm})$, i.e., the elements belonging to the nerve, according to $[16,17]$, are extracted. Analytically,

$$
\begin{gathered}
\gamma_{B}=\Gamma \circ B=\cup\left\{(B)_{z} \mid(B)_{z} \subseteq \Gamma\right\} \\
\overline{\gamma_{B}}=\Gamma \backslash \gamma_{B}=\left\{\mathbf{x} \in \Gamma \mid \mathbf{x} \notin \gamma_{B}\right\}
\end{gathered}
$$

where $B$ is the structuring element and $B_{z}$ denotes its translation. The top-hat operation is denoted with the overline.

Since these residual objects can be considered a set of points, the outliers of a mathematical quadratic distribution can be discarded by means of the 


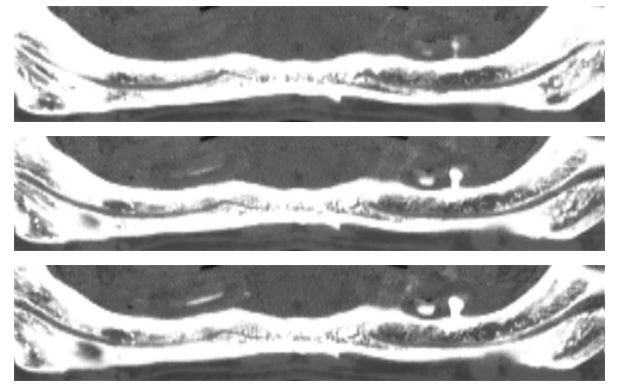

a)

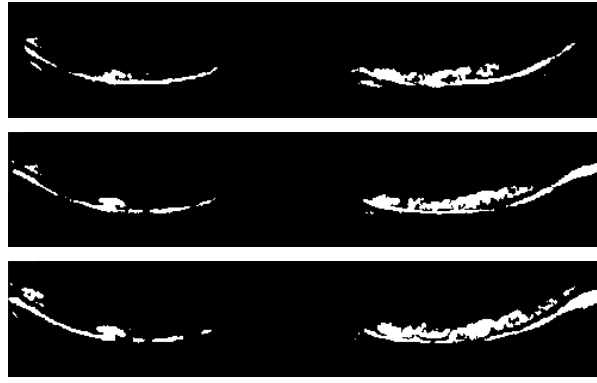

b)

Figure 6: a) Consecutive projections containing the inferior alveolar nerve; b) Inner part of the cortical bone of the same projections after thresholding.

RANSAC algorithm [18]. The remaining points (fig.7.b) are considered to be inliers of the distribution and are assumed to be part of the nerve; therefore, they can be fitted with a quadratic polynomial. These points constitute a

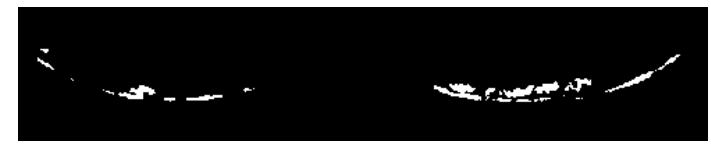

a)

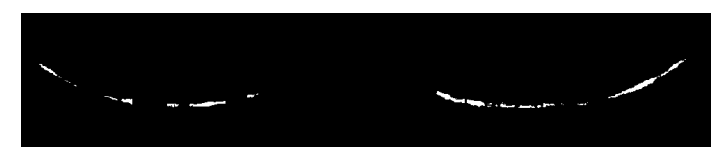

b)

Figure 7: a) AND operation of three consecutive thresholded projections; b) Set of inliers or a quadratic distribution filtered with RANSAC.

system with many more equations than unknowns, that is, an overdetermined system that can be easily solved by the least squares method. Figure 8 shows an example of an estimated curve that is superimposed on its corresponding pseudo-orthopantomographic projection. The coordinates of the points that constitute the curve are used as seeds of the segmentation method.

However, in those areas where the nerve is not distinguishable, the FCOE is not able to delimit the area by the nerve. In these cases, the algorithm finds paths to exit the tissue, and for this reason, it classifies areas belonging to the trabecular bone as the nerve. With the pseudo-orthopantomagraphic projections, it is possible to detect a priori those cross-sections where the FCOE algorithm will possibly lead to a wrong segmentation (from now on, referred to as unlikely-success cross-sections). To this end, the same three consecutive projections are overlapped with an OR operation to get the worst-case 


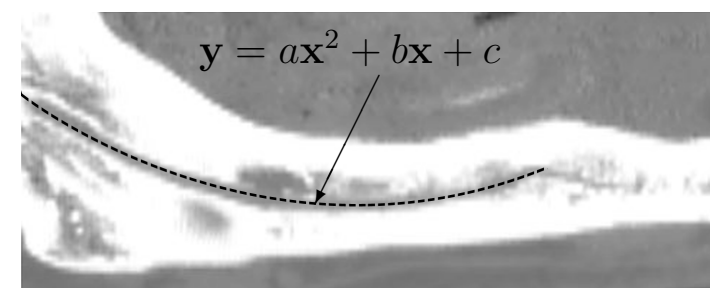

Figure 8: Fitting curve for estimating seeds of FCOE

scenario (fig.9.a).

$$
\Gamma_{w c}=\Gamma_{t h}^{i-1} \cup \Gamma_{t h}^{i} \cup \Gamma_{t h}^{i+1}
$$

The same top-hat operation is performed, and the resulting image (fig.9.b) shows those cross-sections that are suitable for FCOE. In the rest of the set, only the hard tissues segmented. Since the nerve cannot be segmented, it is predicted.

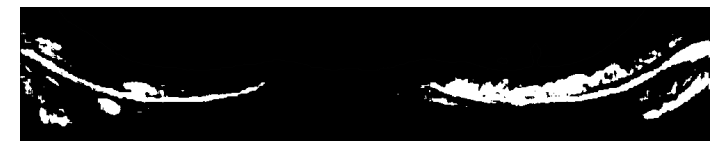

a)

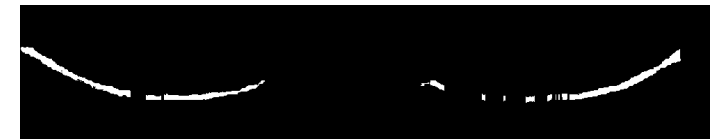

b)

Figure 9: a) OR operation of three consecutive thresholded projections; b) Set of inliers or a quadratic distribution filtered with RANSAC.

\subsection{Analysis and processing}

As stated above, each cross-section $\Omega_{i}$ is analyzed and processed according to the region it belongs to, and the tissues present in it are segmented when possible. In those slices where the nerve is present but undistinguishable, the nerve is predicted. Once the whole set is processed, the different tissues are reconstructed and the 3D volume is obtained.

To facilitate understanding of the proposed method, the algorithm has been divided into three fundamental steps: hard tissues processing, nerve processing and trabecular core processing as shown in figure 10. All the blocks are explained in their respective subsections and are detailed in figure 11. 


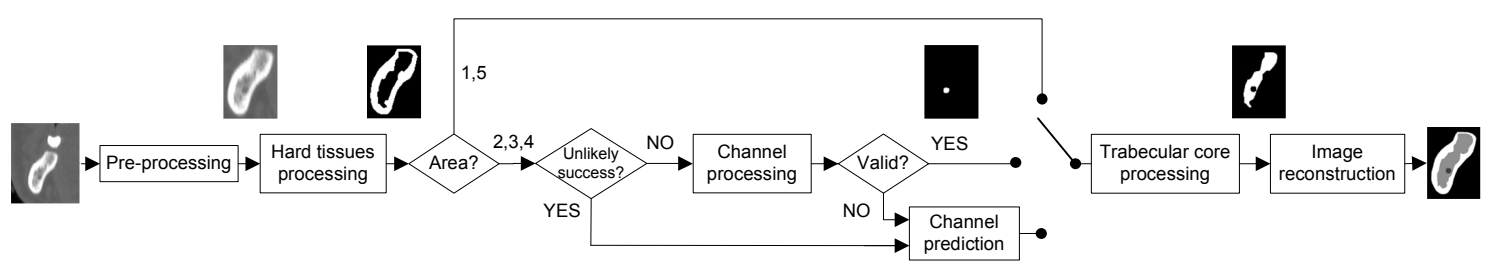

Figure 10: Flowchart of the processing algorithm.

In order to improve the computational cost of the algorithm, only the region of interest(ROI) of each cross-section is considered. The ROI is computed as the area covered by the hard tissues, adding a safety margin. This task is carried out in the preprocessing step shown in figure 10.As this figure shows, the hard tissues are segmented into their corresponding block as explained in subsection 2.3.1. Depending on the area that the cross-section belongs to, it is processed in different ways. If the cross-section belongs to an area where the channel is not present, that is areas 1 and 5, the channel is not evaluated and the trabecular core is defined as the inner part of the cortical bone.

On the other hand, if the cross-section belongs to an area where the channel is present (i.e. areas 2, 3 and 4), the channel must be properly defined as described in subsection 2.3.2. If the cross-section under study is an unlikely-success slice, the inferior alveolar channel is predicted as explained in subsection 2.4. However, if the segmentation of the channel is a priori plausible, the tissue is defined by means of the FCOE algorithm. If the segmented channel has some required properties, it is considered to be a valid channel. Otherwise, the channel is also predicted. Finally, the trabecular core is defined as the inner part of the cortical bone that is not considered to be part of the channel, as described in subsection 2.3.3.

\subsubsection{Hard tissues processing}

The hard tissues are defined by directly thresholding on Hounsfield values. Worse results with higher computational cost have been obtained with other segmentation methods [10]. The experimental results show that cortical bone and teeth have density values of about $+800 \mathrm{HU}$, but this value will clearly vary depending on the patient's anatomy and the configuration of the CT scanner. In these cases, this parameter can be tuned manually.

To be coherent with the real anatomy of the jaw, a boundary is added 


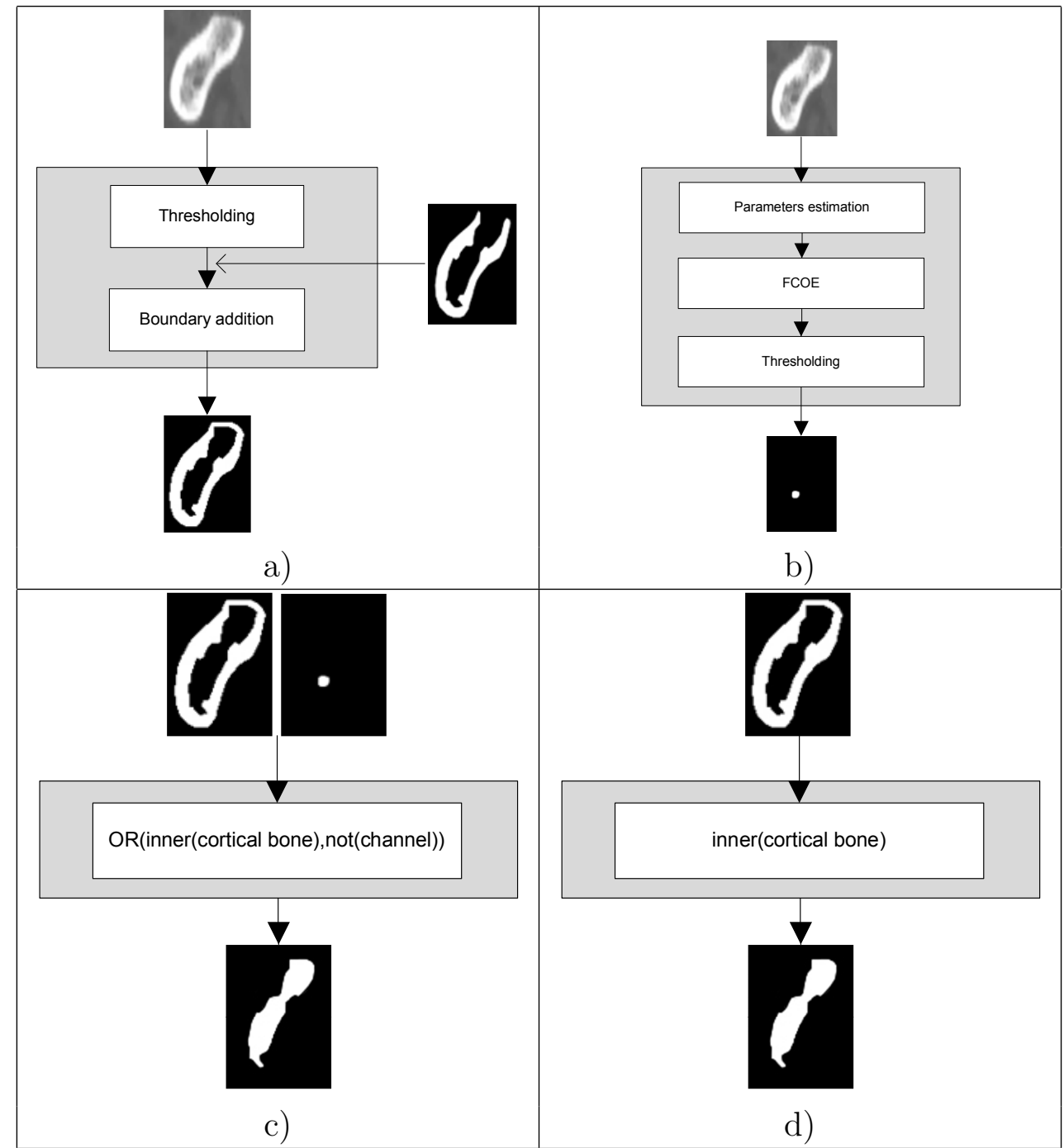

Figure 11: Processing flowchart of a) cortical bone; b) nerve; c) and d) trabecular core.

in each cross-section, as shown in figure 11.a so that this tissue is always represented by a closed surface. The boundary is estimated by means of morphological operations as the residue between the section of the hard tissues and an erosion of it with a small structuring element. The section of the hard tissues is the result of the morphological closing of the cross-sectional image with a structuring element large enough to remove the darkest inner 
areas of the cortical bone. The algorithm 2 shows this process.

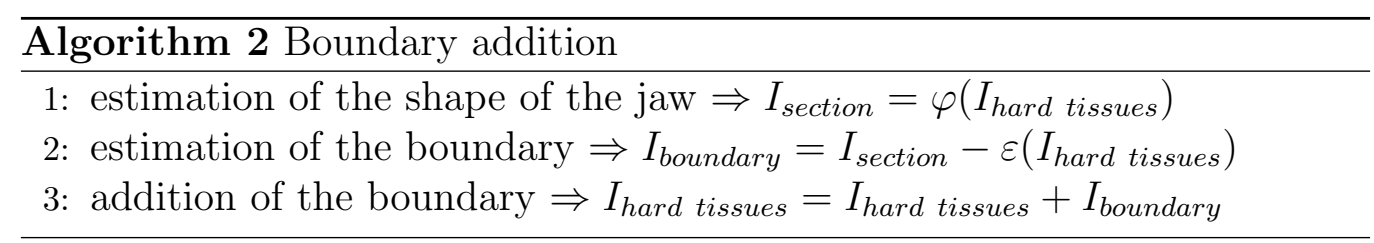

The expression $\varepsilon(I)$ represents the morphological erosion of image $I$.

\subsubsection{Channel processing}

First, the surroundings of the seed are analyzed to obtain the parameters needed by the FCOE algorithm. Then, the algorithm computes the connectivity map of the tissue, which is thresholded and transformed into a binary image. This process is depicted in figure 11.b. Afterwards, the blobs are analyzed and forced to fulfill some requirements to be considered a plausible channel, as deduced from $[16,17]$.

- channel.area $\geq 1.54 \mathrm{~mm}^{2}$ \& channel.area $\leq 14.52 \mathrm{~mm}^{2}$

- $\mid$ channel.centroid - lastchannel.centroid $\mid<1 \mathrm{~mm}$

- $\mid$ channel.area - lastchannel.area $\mid<1.35 \mathrm{~mm}^{2}$

Any blob with a size that is coherent with these values and does not have significant variations as regards previous cross-sections $(1 \mathrm{~mm} / 4$ pixels related to centroid and $1.35 \mathrm{~mm}^{2} / 20$ pixels related to area) is considered to be a valid channel and is therefore saved. If, on the contrary, the blob does not fulfill the requirements, it is rejected and predicted as described in section 2.4.

\subsubsection{Trabecular core processing}

The definition of the trabecular core depends on the area that the crosssection belongs to, as shown in figure 11.c and 11.d. In areas 1 and 5 (where the nerve is not present), the trabecular core is defined as the inner part of the cortical bone, hence the need for this tissue to be a closed surface. However, in areas 2, 3 and 4 (where the nerve is present), the trabecular core is defined as the inner part of the cortical bone, that does not belong to the nerve. 


\subsection{Gap filling}

The nature of human tissues prevents the segmentation of some crosssections in the processing step. Similar density values of trabecular bone and nerve induce similar grayscale values, in such a way that the reliable segmentation of the nerve is not possible. This fact leads to the appearance of segmentation gaps in the cross-sectional set, as shown in figure 12 .

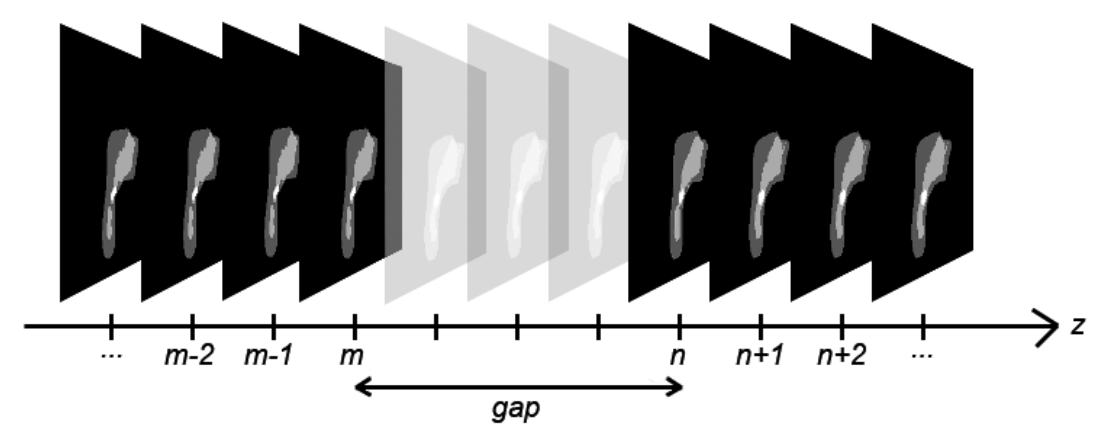

Figure 12: The inability to segment some slices leads to the appearance of gaps among the cross-sections.

In order to obtain the 3D volume of the tissues under study, the reconstruction process requires all the cross-sections to be segmented and thus the need for filling the gaps in the segmented set. To achieve this goal, an interpolation technique using Fourier descriptors is implemented, as in $[19,20]$. The hard tissues are easily segmented by thresholding, even in the problematic slices, and the trabecular core can be extracted as described in section 2.3.3; therefore our efforts are focused on recovering the nerve in those cases. The contour of $2 \mathrm{D}$ regions in the cross-sectional views of the inferior alveolar nerve on the border slices of every gap ( $m$ and $n$ in figure 12) can be described by a finite set of points. Their coordinates can be used to denote them as complex numbers and then the Fourier transform (FT) can be applied to define the Fourier descriptors as follows:

$$
Z_{t}[k]=\frac{1}{N} \sum_{n=0}^{N-1} z[n] e^{-j(2 \pi / N) k n}
$$

where $t$ identifies the cross-section, and $N$ is the smaller power of two that is larger than the maximum of the number of points of both border slices. 
The cross-sections in between are linearly predicted as shown in equation 12 . Non-linear techniques would lead to an undesirable acceleration in the transformation of the shape. However, as figure 8 shows, since the nerve describes an almost quadratic path along the hemimandible, the first Fourier descriptor (for $\mathrm{k}=0$ ), which represents the mass center of the shape, is predicted by means of a quadratic polynomial. The rest of the coefficients are estimated as follows:

$$
Z_{t}[k]=\left(1-\frac{t}{T}\right) Z_{m}[k]+\frac{t}{T} Z_{n}[k]
$$

Afterwards, the high frequency of Fourier descriptors is filtered in order to acquire a smoother boundary. The experimental results show that significant descriptors occupy lower (normalized) frequencies than 0.05. Finally, the boundary points of each cross-section can be easily recovered by applying the inverse of the Fourier transform. The whole process is shown in figure 13.

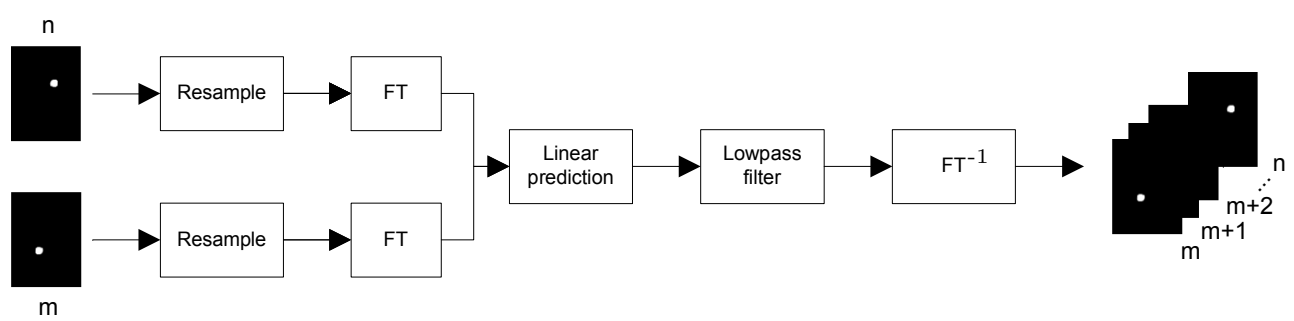

Figure 13: Gap filling flowchart

An example of predicted missing cross-sections is shown in Figure 14. This figure shows the evolution of the boundary from the initial (first frame) to the final shape (last frame). Thirteen intermediate shapes were used for the transformation.

\section{5. $3 D$ reconstruction}

To shape the 3D volume of the segmented tissues, the marching cubes algorithm is used as described in [21]. The algorithm locates the surface in a virtual cube created from eight pixels, which constitute the eight vertices of the cube (four each from two adjacent cross-sections). The algorithm then determines how the surface intersects the cube, and goes on to the next cube, and so on. This is why the segmentation of the whole set of cross-sections is so important. According to the configuration of the pixels, one of 256 

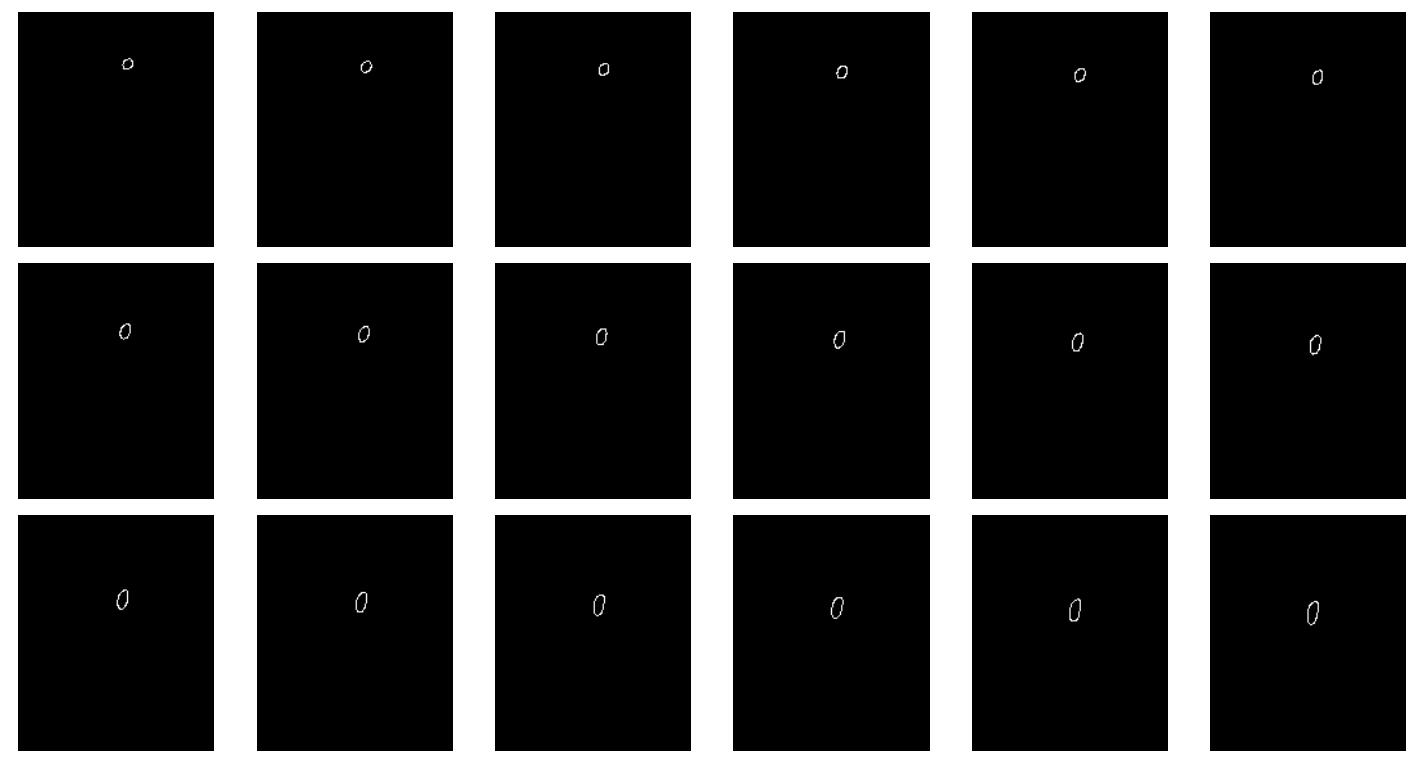

Figure 14: Prediction of intermediate shapes using Fourier descriptors

predefined surfaces is assigned to each cube shaping the surface. Figure 15 shows an example of a 3D reconstruction of a patient's jaw. 


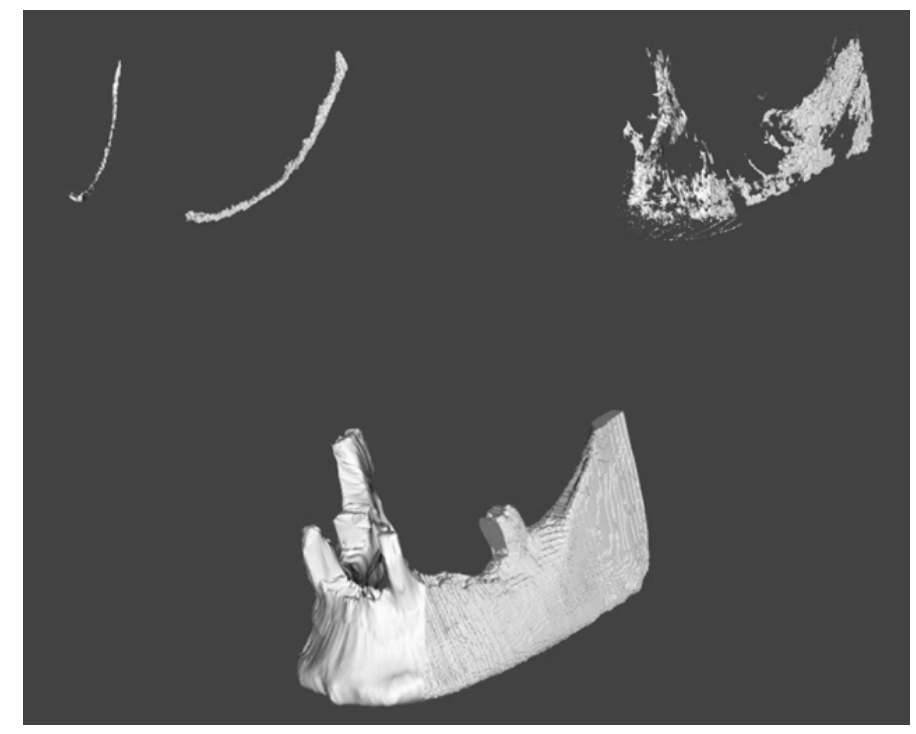

Figure 15: Reconstructed 3D volume of inferior alveolar nerve (upper left), trabecular core (upper right) and hard tissues (bottom) from segmented cross-sections

\subsection{Data Analysis}

In order to evaluate the accuracy of the segmentation process, $20 \mathrm{CT}$ studies from 20 different patients were considered. A group of five experts (dentists and surgeons) was involved in the segmentation process. To constitute the groundtruth, the experts manually segmented 20 hemimandibles, on which some intervention was desired to be planned. The hemimandibles consisted of 404 to 482 cross-sections, so the experts segmented by general consent about 9000 cross-sections, which is an extremely arduous task. Nevertheless, this task allows us to analytically evaluate the results of the segmentation process, by using 4 different indicators of similarity and comparing the cross-sections segmented by the proposed method with the groundtruth. The indicators used were the Jaccard index, Dice's coefficient, point-to-point and point-to-curve distances, all of which are defined below, where suffixes seg and $g t$ refer to segmented and groundtruth images, respectively.

- Jaccard index (JI)

$$
J I\left(I_{\text {seg }}, I_{g t}\right)=\frac{I_{\text {seg }} \cap I_{g t}}{I_{\text {seg }} \cup I_{g t}}, \text { where } 0 \leq J I \leq 1
$$


- Dice's coefficient (DC)

$$
D C\left(I_{\text {seg }}, I_{g t}\right)=\frac{2\left|I_{\text {seg }} \cap I_{g t}\right|}{\left|I_{\text {seg }}\right|+\left|I_{g t}\right|}, \text { where } 0 \leq D C \leq 1
$$

- Point-to-point distance (PPD)

$$
P P D\left(I_{\text {seg }}, I_{g t}\right)=\frac{1}{n} \sum_{i=1}^{n} \sqrt{\left(x_{\text {seg }}^{i}-x_{g t}^{i}\right)^{2}+\left(y_{\text {seg }}^{i}-y_{g t}^{i}\right)^{2}}
$$

where $x$ and $y$ denote the two coordinates of every point, and $n$ denotes the number of points established along the surface of both shapes.

- Point-to-curve distance (PCD)

$$
P C D\left(I_{\text {seg }}, I_{g t}\right)=\frac{1}{n} \sum_{i=1}^{n} \min _{t} \sqrt{\left(x_{\text {seg }}^{i}-r_{y}(t)\right)^{2}+\left(y_{\text {seg }}^{i}-r_{x}(t)\right)^{2}}
$$

where $r(t)=\left(r_{x}(t), r_{y}(t)\right), t \in[0,1]$ denotes a linear spline defined in the groundtruth image, as described in [22].

\section{Results}

The cross-sectional sets of the 20 patients were measured with the 4 aforementioned similarity indicators, and the mean values obtained and standard deviation for all the cases are shown in table 3 .

\begin{tabular}{|l|l|l|l|l|}
\hline & JI & DC & PPD & PCD \\
\hline Mean & 0.7259 & 0.8396 & $0.1440 \mathrm{~mm}$ & $0.1628 \mathrm{~mm}$ \\
\hline Standard deviation & 0.0308 & 0.0187 & $0.0230 \mathrm{~mm}$ & $0.0246 \mathrm{~mm}$ \\
\hline
\end{tabular}

Table 3: Mean values for the analysed indicators

Figure 16 shows two examples of segmented patients. The hard tissues, the trabecular core and the inferior alveolar nerve are positioned with the same spatial orientation to facilitate the interpretation. 

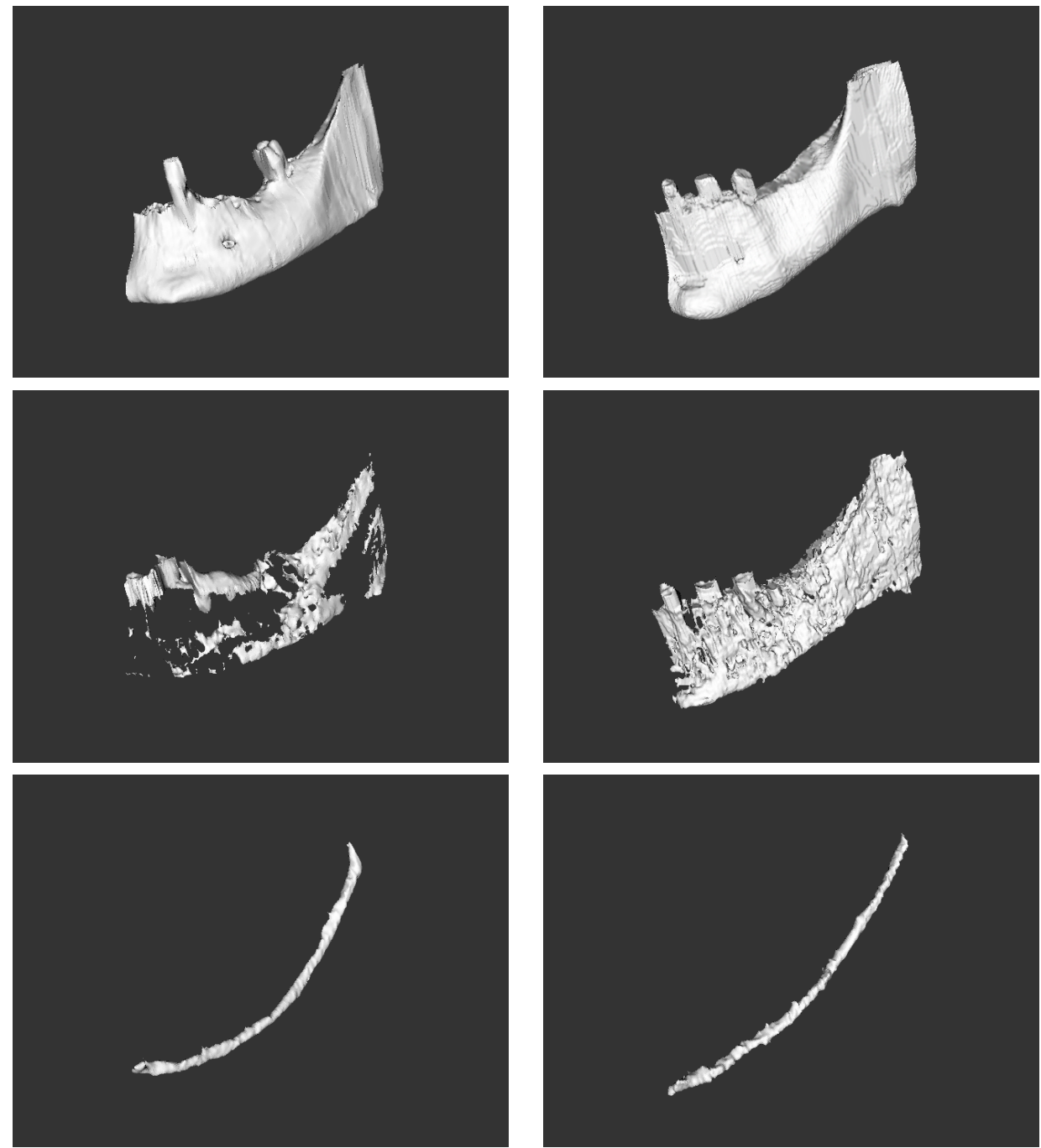

Figure 16: Figure 16 shows two examples of segmented patients. The hard tissues, the trabecular core and the inferior alveolar nerve are positioned with the same spatial orientation to facilitate the interpretation.

An example of the mesh resulting from the marching cubes algorithm is shown in figure 17. This figure shows the path of the inferior alveolar nerve along the jaws of two patients, focusing on the exit of this tissue through the mental foramen. The mental loop can be easily observed in the images. 

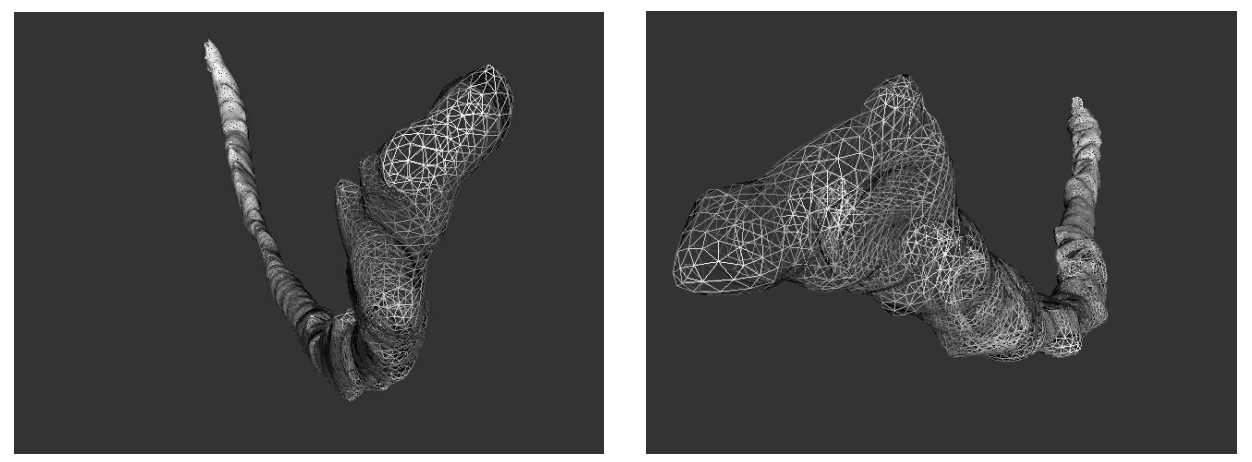

Figure 17: The mental loop is represented with high accuracy as shown in the marching cubes mesh

\section{Discussion}

Since an inferior alveolar nerve injury can lead to different complications, its location must be known to carry out any surgery that affects its surrounding structures. However, the similarity in the density values of the jaw tissues complicates its segmentation. Several approaches have been presented aiming this goal with significant differences among them, which proves that there is still no clinical agreement in the way to achieve it. This manuscript describes a new approach based on mathematical morphology and on the fuzzy connectedness object extraction. Since the segmentation process is performed in cross-sectional images, the evaluation of the method has focused on studying the $2 \mathrm{D}$ segmentation in the cross-sections that define the path of the nerve along the jaw. In other words, the evaluation strategy is based on the comparison of processed images with other previously segmented images, known as the groundtruth set. There are no public databases of segmented dental CT, which led us to manually segment different studies to generate our own groundtruth set. Five experts carried out an extremely heavy task, segmenting manually more than 9000 cross-sections from 20 different patients, and defining a significant database compared to other methods [13].

The absence of databases with normalized segmented dental CT also prevents the comparison with other similar methods and the standardization of the results of each method. In addition, radically different strategies are used by different authors, which make this generalization even more difficult.

The results of the presented method in the described dataset have been promising in all the considered similarity indicators. Regarding the overlap 
indicators, the method has achieved high values in the processed dataset, that is, $0.726 \pm 0.031$ and $0.840 \pm 0.002$ (in terms of mean and standard deviation) in the $J I$ and $D C$ indicators, respectively. Those values are remarkable considering that these indicators range from 0 to 1 , where 0 represents the absence of common areas and 1 represents a perfect match. Our results are even significantly higher than the $\mathrm{DC}$ threshold value $(D C=0.7)$ from which an overlap is commonly considered good [23, 24]. Regarding the distance indicators ( $P P D$ and $P C D$ ), the method has achieved significant results in accuracy. Specifically, the point-to-point distance and point-tocurve distance have been $0.144 \pm 0.023 \mathrm{~mm}$ and $0.163 \pm 0.025 \mathrm{~mm}$ (also in terms of mean and standard deviation), respectively, which are remarkable values taking into account that the diameter of the nerve is approximately $2.5 \mathrm{~mm}[16,17]$. Considering this diameter, the mean errors of both measures are, expressed as percentages, 5.76 and $6.52 \%$, respectively. The presented results show that our method obtains higher accuracy values when compared with other state-of-the-art methods [9, 13].

The configuration of the morphology-based and fuzzy connectednessbased algorithms used in the presented validation is provided in the corresponding subsections. In all the cases, experimental results showed that 8-adjacency provided very similar results than 4-adjacency while significantly increased the computational cost. This can be due to the recurring nature of the fuzzy connectedness algorithm which ends up computing the significant areas of the image, independently of the adjacency.

With regards to the input data used, in spite of the fact that the method is presented for CT data, it should be easily adaptable to Cone Beam Computed Tomography (CBCT) data, since images derived from data obtained by dental cone beam tomographs are substantially similar to dental CT. This fact should be taken into account due to the increasing number of CBCT systems that are being incorporated in the clinical settings. In addition, since no special considerations are needed and no particular orientation of the patient is required during the CT scans, the thyroid gland and patient's lens do not have to undergo unusual doses of radiation, thus avoiding higher cancer and posterior subcapsular cataract risk [25].

Although the presented method has focused on the jaw tissues, it can be easily extendible to maxillary cases, since the maxilla can be interpreted as an area where the nerve is not present, or even to other body structures with similar tubular shape. 


\section{Conclusions and future work}

This paper has presented a new method for the segmentation and reconstruction of tissues of the human jaw. The method consists of sequentially segmenting cross-sections that are defined perpendicularly to the dental arch plane, starting from a CT volume of the patient and then reconstructing the tissues using the marching cubes algorithm. The algorithm has been exhaustively measured for four indicators: the Jaccard index, Dice's coefficient, and point-to-curve and point-to-point distances, achieving a high level of accuracy and providing reliable information to computer-aided programs in order to facilitate oral surgery.

Future work will focus on adapting the algorithm in $\mathrm{C}++$ language using ITK and VTK libraries in order to increase the computational speed towards a real time application. Further testing of other segmentation methods is also needed in order to improve accuracy. Due to the random nature of the RANSAC algorithm, other ways of isolating the path of the mandibular canal must also be tested.

[1] K. Verstreken, J. V. Cleynenbreugel, K. Martens, G. Marchal, D. van Steenberghe, P. Suetens, An image-guided planning system for endosseous oral implants, IEEE Transactions on Medical Imaging 17 (5) (1998) 842-852.

[2] C. C. Galanis, M. M. Sfantsikopoulos, P. T. Koidis, N. M. Kafantaris, P. G. Mpikos, Computer methods for automating preoperative dental implant planning: Implant positioning and size assignment, Computer Methods and Programs in Biomedicine 86 (1) (2007) 30-38.

[3] S. Fütterling, R. Klein, W. Straßer, H. Weber, Automated finite element modeling of a human mandible with dental implants, in: 6th International Conference in Central Europe on Computer Graphics and Visualization, 1998.

[4] P. Kršek, P. Krupa, P. Cernochová, Teeth and jaw 3d reconstruction in stomatology, in: Medical Information Visualization - BioMedical Visualization, IEEE Computer Society, 2007, pp. 23-28.

[5] C. Xiaojun, Y. Ming, L. Yanping, W. Yiqun, W. Chengtao, Image guided oral implantology and its application in the placement of zygoma implants, Computer Methods and Programs in Biomedicine 93 (2) (2009) 162-173. 
[6] W. Stein, S. Hassfeld, J. Muhling, Tracing of thin tubular structures in computer tomographic data, Computer Aided Surgery 3 (2) (1998) $83-88$.

[7] T. Kondo, S. Ong, K. W. Foong, Computer-based extraction of the inferior alveolar nerve canal in 3-d space, Computer methods and programs in biomedicine 76 (3) (2004) 181-191.

[8] S. Rueda, J. A. Gil, R. Pichery, M. A. niz, Automatic segmentation of jaw tissues in ct using active appearance models and semi-automatic landmarking, Medical Image Computing and Computer-Assisted Intervention MICCAI 20064190 (2006) 167-174.

[9] D. Kainmueller, H. Lamecker, H. Seim, M. Zinser, S. Zachow, Automatic extraction of mandibular nerve and bone from cone-beam ct data, in: Medical Image Computing and Computer-Assisted Intervention MICCAI 2009, Vol. 5762, 2009, pp. 76-83.

[10] R. Lloréns, V. Naranjo, M. Clemente, M. Alcañiz, S. Albalat, Validation of fuzzy connectedness segmentation for jaw tissues, in: Bioinspired Applications in Artificial and Natural Computation, Springer Berlin / Heidelberg, 2009, pp. 41-47.

[11] J. K. Udupa, S. Samarasekera, Fuzzy connectedness and object definition, In SPIE Proceedings Medical Imaging 2431 (1995) 02-10.

[12] J. Udupa, S. Samarasekera, Fuzzy connectedness and object definition: Theory, algorithms, and applications in image segmentation, Graphical Models and Image Processing 58 (3) (1996) 246-261.

[13] G. Kim, J. Lee, H. Lee, J. Seo, Y.-M. Koo, Y.-G. Shin, B. Kim, Automatic extraction of inferior alveolar nerve canal using feature-enhancing panoramic volume rendering, Biomedical Engineering, IEEE Transactions on 58 (2) (2011) $253-264$.

[14] P. K. Saha, J. K. Udupa, D. Odhner, Scale-based fuzzy connected image segmentation: Theory, algorithms, and validation, Computer Vision and Image Understanding 77 (2000) 145-174.

[15] J. Serra, Image analysis and mathematical morphology, Academic Press, London, 1982. 
[16] G. M. Reiser, J. D. Manwaring, P. D. Damoulis, Clinical significance of the structural integrity of the superior aspect of the mandibular canal, Proceedings of the 3rd International Work-Conference on The Interplay Between Natural and Artificial Computation: Part II: Bioinspired Applications in Artificial and Natural Computation 5602 (2009) 11-19.

[17] I. C. Suazo, C. A. Morales, M. G. Cantn, D. A. Zavando, Biometric aspects of the mandibular canal, International Journal of Morphology 25 (4) (2007) 811-816.

[18] M. A. Fischler, R. C. Bolles, Random sample consensus: a paradigm for model fitting with applications to image analysis and automated cartography, Communications of the ACM 24 (6) (1981) 381-395.

[19] F. Marqués, B. Llorens, A. Gas, Prediction of image partitions using fourier decriptors: application to segmentation-based coding schemes, IEEE Transactions on image processing 7 (4) (1998) 529542.

[20] F. Marqués, B. Llorens, A. Gasull, Interpolation and extrapolation of image partitions using fourier descriptors: application to segmentationbased coding schemes, Image Processing, International Conference on 3 (1995) 584-587.

[21] W. E. Lorensen, H. E. Cline, Marching cubes: A high resolution 3d surface construction algorithm, in: SIGGRAPH '87: Proceedings of the 14th annual conference on Computer graphics and interactive techniques, Vol. 21, 1987, pp. 163-169.

[22] M. B. Stegmann, Active appearance models: Theory, extensions and cases (2000).

[23] K. H. Zou, S. K. Warfield, A. Baharatha, C. Tempany, M. R. Kaus, S. J. Haker, W. M. Wells, F. A. Jolesz, R. Kikinis, Statistical validation of image segmentation quality based on a spatial overlap index, Academic Radiology 11 (2004) 178-189.

[24] A. Zijdenbos, B. Dawant, R. Margolin, A. Palmer, Morphometric analysis of white matter lesions in mr images: method and validation, Medical Imaging, IEEE Transactions on 13 (4) (1994) 716 -724. 
[25] P. L. Kaufman, F. H. Adler, A. Alm, Adler's Physiology of the Eye, 10th Edition, Elsevier, 2003. 